\title{
On classical description of radiation from neutral fermion with anomalous magnetic moment
}

\author{
A.E. Lobanov and O.S. Pavlova * \\ October 30, 2018 \\ Moscow State University, \\ Department of Theoretical physics. \\ 117234, Moscow, Russia
}

\begin{abstract}
Electromagnetic radiation from an uncharged spin $1 / 2$ particle with an anomalous magnetic moment moving in the classical electromagnetic external field originates from quantum spin-flip transitions. Although this process has a purely quantum nature, it was observed for certain particular external field configurations that, when quantum recoil is neglected, the radiation power corresponds to the classical radiation from an evolving magnetic dipole. We argue that this correspondence has a more general validity in the case of an unpolarized particle and derive a general formula for radiation in terms of the external field strength and its derivative. A classical dynamics of the spin is described by the Bargmann-Michel-Telegdi equation.
\end{abstract}

PACs: 03. 65. Sq

Electromagnetic radiation from an uncharged spin $1 / 2$ particle possessing an anomalous magnetic moment moving in an external classical electromagnetic field was studied in a number of papers [1-8]. The usual approach consists in a computation of the radiative transitions within the Furry's picture of quantum electrodynamics (QED). This appeals to the knowledge of exact solutions of the Dirac equation with an anomalous magnetic moment in the external electromagnetic field. Such solutions are known for various particular configurations of the electromagnetic field: homogeneous fields [1,2], plane-waves [3-6], and some other [7,8], for them an explicit computation of radiative transitions was performed. Radiative transitions in the case of the uncharged particles correspond to the spin-flip amplitudes and thus have an essentially quantum nature. Meanwhile it was observed that under certain conditions the radiation process may be described in purely classical terms using the Bargmann-Michel-Telegdi (BMT)

*E-mail: OSPavlova@aport.ru 
spin evolution equation $[9,10]$. In such a pseudoclassical treatment the radiation power is given by the well-known formula for radiation from a magnetic moment $\mu^{\nu}[11]$ :

$$
\frac{d I}{d \mathcal{O}}=-\frac{1}{4 \pi(l u)^{5} u^{0}}\left\{\ddot{\mu}^{\nu} \ddot{\mu}_{\nu}(u l)^{2}+\left(l^{\nu} \ddot{\mu}_{\nu}\right)^{2}\right\}
$$

where $(u l)=u^{\nu} l_{\nu}, u^{\nu}$ is the 4 -velocity of a particle, $l^{\nu}=\{1,1\}, \mathbf{l}$ is the unit vector in the direction of an emitted wave; a dot denotes the differentiation with respect to the proper time $\tau$. We use the units $\hbar=c=1$.

The radiation power calculated within the framework of QED was found to correspond to the result obtained from the equation (1) under the following conditions. The particle motion has to be quasiclassical, in a sense that:

i) the binding energy due the magnetic moment in the rest frame is much smaller than the particle mass (here we use gaussian units):

$$
H_{0} \ll H_{c r}, \quad H_{c r}=m^{2} c^{3} / e \hbar,
$$

( $H_{0}$ is the magnetic field strength in the rest frame),

ii) the external field varies slowly at the distances of the order of a Compton's length, which amounts to conditions:

$$
\hbar \dot{H}_{0} / m c^{2} H_{0} \ll 1, \quad\left(H_{0} / H_{c r}\right)\left(\omega / \omega_{c}\right) \ll 1,
$$

(here $\omega$ is the characteristic frequency change of the external field, and $\omega_{c}=e H / m c$ is the cyclotron frequency).

If one is interested in the radiation from an unpolarized particle, an additional requirement to be imposed consists in averaging over the initial spin states and summing over the final polarizations. It can be expected that the averaging of the quantum transition amplitudes should correspond to the averaging over the initial orientation of the magnetic dipole moment within the classical picture.

Although no general derivation of such a pseudoclassical description from QED is available for the case of an arbitrary external field, it seems very plausible that under the above assumptions the result should follow indeed from the classical formula (1). Our conjecture is that one has to replace the magnetic moment by the quantity

$$
\mu^{\nu}=\mu_{0} S^{\nu}
$$

where $S^{\nu}$ is expectation value of the spin vector, and perform an averaging over the polarizations at $\tau=\tau_{0}$ :

$$
\begin{gathered}
\frac{d I}{d \mathcal{O}}=-\frac{\mu_{0}^{2}}{4 \pi(l u)^{5} u^{0}}<\left\{\ddot{S}^{\mu} \ddot{S}_{\mu}(u l)^{2}+\left(l^{\mu} \ddot{S}_{\mu}\right)^{2}\right\}>= \\
=-\frac{\mu_{0}^{2}}{4 \pi(l u)^{5} u^{0}}<e^{\mu \nu \rho \lambda} \ddot{S}_{\nu} u_{\rho} l_{\lambda} e_{\mu \nu^{\prime} \rho^{\prime} \lambda^{\prime}} \ddot{S}^{\nu^{\prime}} u^{\rho^{\prime}} l^{\lambda^{\prime}}>
\end{gathered}
$$

An evolution of the classical spin is described by the BMT equation [12]:

$$
\dot{S}^{\nu}=2 \mu_{0}\left\{F^{\nu \alpha} S_{\alpha}-u^{\nu}\left(u_{\alpha} F^{\alpha \beta} S_{\beta}\right)\right\},
$$


whose solution has to be constrained by the conditions $S^{\nu} S_{\nu}=-1, \quad S^{\nu} u_{\nu}=0$. The validity of this equation is ensured by the conditions (2), (3) [13, 14]. Our main goal is to show that when the averaging over polarization states is performed the resulting expression for the radiation power will depend only on the external field intensity and thus will be valid in the case of arbitrary external field subject to conditions (2),(3). We check that it is true indeed for the particular fields studied before.

An important point in the derivation is that the neutral particle moves in the external field with a constant velocity. Of course, the true quantum description of radiation demands that the quantum recoil in the photon emission process should be taken into account. But when conditions (2), (3) are satisfied, energy of emitted photon is small, therefore we can neglect the recoil, i.e. the change of particle velocity. This assumption was made in the formula (5). In the BMT equation one can pass to the rest frame of the particle where the required averaging over polarizations can be easily performed.

It is more convenient to rewrite the BMT equation in term of the $S L(2, \mathbf{C})$ spintensors. Instead of the 4-vector $S^{\nu}$ we will use matrices $S=S^{0} \sigma_{0}+\boldsymbol{S} \boldsymbol{\sigma}$ and $\tilde{S}=$ $S^{0} \sigma_{0}-\boldsymbol{S} \boldsymbol{\sigma}$, where $\sigma_{\nu}$ are the Pauli matrices (we follow a notation of [15]).

Then the evolution of spin is given by the following matrix operator [16]:

$$
\underset{\sim}{S}(\tau)=\underset{\sim}{\operatorname{Lr}} \underset{\sim}{L_{\sim}^{-1}} \underset{\sim}{S_{0}}\left(\underset{\sim}{\left.L^{-1}\right)^{+}} \underset{\sim}{R^{+}} \underset{\sim}{L^{+}}\right.
$$

Here $S_{0} \equiv S\left(\tau_{0}\right)$ is the polarization at the initial moment of time $\tau_{0}, \underset{\sim}{L^{-1}}$ is a transformation to the particle rest frame

$$
\underset{\sim}{L^{-1}}=\frac{1+\tilde{u}}{\sqrt{2\left(1+u^{0}\right)}}, \quad \underset{\sim}{L}=\frac{1+\underset{\sim}{u}}{\sqrt{2\left(1+u^{0}\right)}}
$$

and $\underset{\sim}{R}$ is a rotation operator satisfying the equation

$$
\underset{\sim}{\dot{R}}=i \mu_{0} \underset{\sim}{H_{0} R}
$$

where

$$
\mathbf{H}_{0}(x) \equiv \mathbf{H}_{0}=u^{0} \mathbf{H}-\mathbf{u} \times \mathbf{E}-\frac{\mathbf{u}(\mathbf{u H})}{1+u^{0}}-
$$

is the magnetic field in the rest frame at the point of the particle location. In this notation the formula (5) can be presented in the form:

$$
\frac{d I}{d \mathcal{O}}=-\frac{\mu_{0}^{2}}{8 \pi(l u)^{5} u^{0}}<S p\left\{\underset{\sim}{\ddot{S}} \ddot{\tilde{S}}(u l)^{2}+\frac{1}{4}[l \underset{\sim}{l} \underset{\tilde{S}}{l} \underset{\sim}{\tilde{S}}+\underset{\sim}{\ddot{S}} \tilde{\sim} \underset{\sim}{\tilde{S}} \tilde{l}]\right\}>
$$

After a simple algebra with account for the Eq.(7) we obtain for the radiation power of unpolarized particle:

$$
\frac{d I}{d \mathcal{O}}=-\frac{\mu_{0}^{2}}{16 \pi(l u)^{3} u^{0}} S p \sum_{i}\left\{\left(\ddot{\sigma_{R}}\right)_{i}\left(\ddot{\sigma_{R}}\right)_{i}-\left(\ddot{\sigma_{R}}\right)_{i}(\boldsymbol{\sigma} \boldsymbol{n})\left(\stackrel{\ddot{\sigma_{R}}}{{ }_{i}}(\boldsymbol{\sigma} \boldsymbol{n})\right\} .\right.
$$


where

$$
\left(\sigma_{R}\right)_{i}=\underset{\sim}{R} \sigma_{i}{\underset{\sim}{2}}^{+}, \quad \mathbf{n}=\frac{1}{(u l)}\left\{\mathbf{l}-\frac{1+(u l)}{1+u^{0}} \mathbf{u}\right\}-
$$

is the unit vector in the direction of radiation in the rest frame of the particle. In (11) the summation over $i$ corresponds to an averaging over the initial spin states and summation over the final spin states.

Using Eqs. (8) and (11) we arrive at the final result

$$
\frac{d I}{d \mathcal{O}}=\frac{\mu_{0}^{4}}{\pi u^{0}(u l)^{3}}\left\{4 \mu_{0}\left\{\mu_{0} \mathbf{H}_{0}^{4}+\mu_{0} \mathbf{H}_{0}^{2}\left(\mathbf{H}_{0} \mathbf{n}\right)^{\mathbf{2}}+\left(\mathbf{H}_{\mathbf{0}} \mathbf{n}\right)\left[\dot{\mathbf{H}}_{\mathbf{0}} \mathbf{H}_{\mathbf{0}} \mathbf{n}\right]\right\}+\dot{\mathbf{H}}_{\mathbf{0}}^{\mathbf{2}}+\left(\dot{\mathbf{H}}_{\mathbf{0}} \mathbf{n}\right)^{\mathbf{2}}\right\} .
$$

Therefore the angle distribution of radiated power is expressed entirely in terms of the magnetic field strength in the rest frame of the particle and its derivative with respect to the proper time.

Formula (12) can be rewritten in a covariant form:

$$
\begin{aligned}
\frac{d I}{d \mathcal{O}}= & \frac{\mu_{0}^{2}}{\pi u^{0}(u l)^{5}}\left\{\left[4\left(\mu_{0}^{2} u_{\rho} H^{\rho \lambda} H_{\lambda \sigma} u^{\sigma}\right)^{2}+\left(\mu_{0}^{2} u_{\rho} \dot{H}^{\rho \lambda} \dot{H}_{\lambda \sigma} u^{\sigma}\right)\right](u l)^{2}\right. \\
& +4\left(\mu_{0}^{2} u_{\rho} H^{\rho \lambda} H_{\lambda \sigma} u^{\sigma}\right)\left(\mu_{0} u_{\rho} H^{\rho \lambda} l_{\lambda}\right)^{2}+\left(\mu_{0} u_{\rho} \dot{H}^{\rho \lambda} l_{\lambda}\right)^{2} \\
& \left.+4 \mu_{0}^{2}\left(\mu_{0} u_{\rho} H^{\rho \lambda} l_{\lambda}\right) e^{\mu \nu \rho \lambda} u_{\mu} \dot{H}_{\nu \sigma} u^{\sigma} H_{\rho \delta} u^{\delta} l_{\lambda}\right\}
\end{aligned}
$$

where $H^{\nu \alpha}=-\frac{1}{2} e^{\nu \alpha \beta \gamma} F_{\beta \gamma}$ is the tensor dual to electromagnetic field tensor.

Integrating over angles, we obtain the formula for the total radiation power:

$$
I=\frac{16}{3} \mu_{0}^{2}\left\{4\left(\mu_{0} \mathbf{H}_{0}\right)^{4}+\left(\mu_{0} \dot{\mathbf{H}}_{0}\right)^{2}\right\}
$$

which has the following covariant counterpart:

$$
I=\frac{16}{3} \mu_{0}^{2}\left\{4\left(\mu_{0}^{2} u_{\rho} H^{\rho \lambda} H_{\lambda \sigma} u^{\sigma}\right)^{2}+\mu_{0}^{2} u_{\rho} \dot{H}^{\rho \lambda} \dot{H}_{\lambda \sigma} u^{\sigma}\right\}
$$

We verified that, substituting into formulas (12), (14), the fields which were earlier used for calculations both by quantum and classical methods, we obtain the full agreement with the results obtained when we neglect the recoil, i.e., terms of the higher order in the Planck constant.

Now let us discuss some possible application for a neutron. An important features of the Eqs. (12) and (14) is the presence of the second term, whose magnitude is defined by the field strength and its derivative. Therefore the radiation power is increased substantially when the boundary of field region which is crossed by a particle is very sharp. This phenomenon similar to transition radiation can be important in astrophysical conditions. Possibly this effect can be observed under the laboratory conditions when fast neutrons pass through the ferromagnetic. 
It is interesting to compare in the order of magnitude the radiation power $I$ from a neutral particle (neutron) and the classical radiation power $I_{0}$ from a charged particle (see, for example, [11]) with the same mass and the energy (proton). We find

$$
\frac{I}{I_{0}} \sim \max \left\{\left(\frac{H_{0}}{H_{c r}}\right)^{2},\left(\frac{u^{0} \hbar \omega}{m c^{2}}\right)^{2}\right\}
$$

Thus, the charge radiation power and the radiation power from a magnetic moment become comparable in very strong fields, or in the fields of high frequency.

\section{Acknowledgments.}

Authors are very grateful to Prof. D. V. Gal'tsov for continuous attention to their work, Prof. A. V. Borisov and Prof. V. Ch. Zhukovsky for fruitful discussions.

\section{References}

[1] I.M. Ternov, V.G. Bagrov, A.M. Khapaev, Zh. Eksp. Teor. Fiz. 48 (1965) 921.

[2] I.M. Ternov, V.G. Bagrov, V.. Kruzhkov, A.M. Khapaev, Izv. Vyssh. Uchebn. Zaved., Fiz. 4 (1967) 30.

[3] V.V. Skobelev, Sov. Phys. JETP 67 (1988) 1322; 68 (1989) 221.

[4] V.A. Bordovitsyn, Izv. Vyssh. Uchebn. Zaved., Fiz. 1 (1971) 131.

[5] I.M. Ternov, V.G. Bagrov, V.A. Bordovitsyn, Ju.A. Markin, Zh. Eksp. Teor. Fiz. 52 (1967) 1584.

[6] A.S. Vshivtsev, R.A. Potapov, I.M. Ternov, J. Exp. Theor. Phys. 78 (1994) 593.

[7] I.M. Ternov, V.G. Bagrov, V.R. Khalilov, Vestn. Mosk. Univ. (3) 2 (1969) 113.

[8] V.G. Bagrov, V.E. Stepanov, Izv. Vyssh. Uchebn. Zaved., Fiz. 6 (1967) 142.

[9] V.A. Bordovitsyn, V.S. Gushchina, Russ. Phys. J. 36 (1993) 247; 37 (1994) 49; 38 (1995) 155; 38 (1995) 293.

[10] V.A. Bordovitsyn, Russ. Phys. J. 36 (1993) 1046.

[11] A.A. Sokolov, I.M. Ternov, V. Ch. Zhukovsky, A.V. Borisov, Quantum electrodynamics, Moscow, Mir (1988).

[12] V. Bargmann, L. Michel, V. Telegdi, Phys. Rev. Lett. 2 (1959) 435.

[13] I.M. Ternov, V.R. Khalilov, O.S. Pavlova, Sov. Phys. J. 21 (1978) 1593; 22 (1979) 140. 
[14] I.M. Ternov, Introduction to Physics of Spin of Relativistic Particles [in Russian], Moscow, Moscow State Univ. (1997).

[15] N.N. Bogolubov, A.A. Logunov, A.I. Oksak, I.T. Todorov, General Principlels of Quantum Field Theory [in Russian], Moscow, Nauka (1980); Engish transl., Kluwer, Dordrecht (1990).

[16] A.E. Lobanov, O.S. Pavlova, Theor. and Math. Phys. 121 (1999) 1691. 